\title{
Formal Perspectives on Conceptualization of Cyberspace
}

\author{
Anas Mu'azu Kademi ${ }^{1}$, Ahmet Koltuksuz ${ }^{2}$ \\ ${ }^{1}$ Yasar University, Turkey, anas.kademi@yasar.edu.tr \\ ${ }^{2}$ Yasar University, Turkey, ahmet.koltuksuz@yasar.edu.tr
}

\begin{abstract}
Cyberization gives rise to divergence of cyberspace concepts- cyberspaces to general cyberspace; cybermatics to cyberSciTech; cyber-enabled worlds to cyber-physical-social-thinking hyperspace etc. That is, Cyberspace perception and conceptualization have become more complex with time, due to scalability and as new terms and ideas are added, leading to continues proliferation creating a need for solid characterization. Existing theories are reach and therefore articulated to better understand the physical social thinking hyperspace notion of cyberspace. This paper presents a meaningful inter-disciplinary, transdisciplinary, and multi-disciplinary integration of cyber philosophy, cyber science, and cyber information to present theoretic perspectives on cyberspace-Formal cyberspace. Three main aspects ontology, topological dynamics, and information respectively considered in terms of philosophy, theory in network science, and information theory to define existence of cyberspatial entity.
\end{abstract}

Key words : Cyber philosophy, Cyber science, Cyberspace, Cyberspace time, Formal cyberspace.

\section{INTRODUCTION}

For many years diverged conception of cyberspace and its related terms persist, with competing definitions from fantasy to spatial cognition- none of which is based on any scientific theory. The boundless use of cyberspace and its related concepts with no consensus is evident as even the way in which cyberspace is written is debatable [1]. This, especially as semantic problem of definition, leads to what Lance Strate called cyberspace(s) [2], and Ning, et al called "General Cyberspace (GC)" [3].

The literature converges at a subset of cyber-physical-social-thinking hyperspace (CPST) through a process of cyberspace evolution called cyberization [4], [5], [6]. A new cyberspace formed from the reformation of cyber-enabled world, substantially alters and revolutionizes the way we conceptualize cyberspace. Fundamentally, cyberspace in which cyber-related elements existence pervade different kind of spaces and disciplines.

The CPST is formed from the merging of cyberphysical systems (CPSs) - a "networked stationary or mobile information systems responsible for the real-time governance of physical processes whose behaviors unfold in cyberspace" [7], and the Internet of things (IoT) [8]. This only emphasizes paradigms intersection of artificial intelligence and cognitive computing covering many areas of applications.

Related studies have also been carried out with respect to the evolutionary dynamics [9], characteristics [4] etc. However, none of these works precisely define cyberspace in relation to the spatial manifolds and existing entities. No formal discussion of philosophy, science, and technology aspects of General Cyberspace, even with the fact that the "space" in cyberspace is significant and a core aspect of its complexity. Space as geometric entity helps to define dimension.

Cyberspace and cyber-enabled spaces are perceived as a consequence of cyberization process, resulting in various field and concepts intersection. For instance, "CyberSciTech" [6], an interdisciplinary integration of cyber science and cyber technology. An emerging interdisciplinary discipline describing cyber entities and behaviors is termed as "Cybermatics" in cyber-physical-social-thinking hyperspace [4], [5], was introduced. Although cybermatics looks promising, it is based on perceptual observation of similar research terms and areas, thus lacking formal connection theme. The work in [11] attempts to link the philosophical concept of cyberspace to cyber science. While the fundamental of philosophy is ontological existence, the contemporary research mainly focuses in aspects of minds, paraspace, social space and ethics.

Most definitions assign an empirical spatial quality to cyberspace though traditional nation of space was abandoned for a meaningful scientific concept. For example, cyberspace "...as the diverse experiences of space associated with computing and related technologies" [2]. In many instances it is perceived in the form of navigation through the space of electronic data, and of control which is achieved by manipulating these data. The 'cyber fiber' [10] and communication channels connect to the physical environment and allow cyberspace users to interact with this real world. Cyberspace's core structure is composed of the interconnected network of all existing communication channels and an information system that connects the computer devices, people and machines.

Cyberspace is completely unprecedented space, but the recent work studies cyberspace analogue to the traditional physical, social and thinking space [13], [14], [15]. The general view is 
that cyberspace is no longer confined to digital world but extends beyond to include various concepts of physical, social and even mental space. Our paper adds a mathematical perspective to the recent studies.

We propose a formalized perspective, a formality of the work in [3], which investigates cyberspace the concepts of existence, interactions, and applications/services in terms of philosophy, science, and technology respectively. In this paper, the Formalization of Cyberspace (FC) is centered around the following: (a) The existence of cyberspatial entities in cyberspace underlined by dimensional conceptarguing on the importance of theory and methodology that characterize cyberspace from ontological viewpoint. (b) Topological mapping, the basics of how the cyberspatial entities are connected; (c) Cyber-informational conjugation, which is concerned with how information entities are conjugated; (d) Cyber Physical-Logical-Information space integration, integrating physical space (Geospace), network space and information space entities.

This paper highlights theoretical foundation of the Generalized Cyberspace based on the views of ubiquitous connections and spaces convergence. It outlined a novel insight on cyberspace and cyber-enabled spaces, and open interesting questions for further research on cyberspace characterization. Specifically, this paper mainly contributes in:

(i) Putting forward the concept of formal cyberspace to include relevant aspects of cyberspace and cyber-enabled spaces in relation to existing spatial theory.

(ii) Interdisciplinary perspective of cyberspace providing a framework for a future research.

(iii) Adding to the functional definition, it highlights the notions of existence, topology and interactions from the perspectives of cyber philosophy, cyber science and cyber information theory.

Following the introduction (section 1), section 2 presents formal and interdisciplinary foundation of formal cyberspace. The existence of spatial theory to explain philosophical ontology of cyberspace and entities is discussed in section 3 . How entities connect to form topology is underlined by a dynamic behavioral pattern, this is presented in section 4 . The conclusion comes in Section 5.

\section{FORMAL CYBERSPACE: DEFINITION AND DISCIPLINES}

Considering the physical basis of cyberspace, there is an overlooked concept of cyberspace rooted in physics. Theories observed in physical space is observed, different from what plausibly discussed [12] but similar to what is implied [13], a three-dimensional view of cyberspace. As an extent in which objects and events have relative position and direction, cyberspace has been broadly defined in [7] as Euclidian space of 9-D hypercube.

Metatheoretical investigation of cyberspace ontological status, we no longer considered cyberspace as paraspace ("an alternate space, sometimes largely mental, but always materially manifested, that sits behind the real world and in which language is raised to an extraordinarily level" [14]). It is important to look beyond the concepts of theoretically unrealized space to establish what is termed as cyberspacetime [2], [7].

\subsection{Disciplines}

\subsubsection{Cyber Philosophy}

A "conceptual issues arising at the intersection of computer science, information science, information technology, and philosophy" [6], Cyber philosophy basically define existence of cyberspatial entities and space, which is an intersection of philosophy and computing [15].

- Ontology: Cyber ontology should address questions such as: "What is cyberspace? Is it or does it have dimension? Are there things in cyberspace? Are things in cyberspace properly called objects? Are such objects or is cyberspace itself substance(s) or process(es)? Is cyberspace or the objects in it real or ideal? What is the categorical scheme of cyberspace? How should cyberspace fit into a broader categorical scheme?" [16]. Our focus is on the first three questions.

- Cyberspacetime: Cyberspace considered as a product of dynamic relationship and geometrically a dimensional manifold with time, is made up of cyberspatial objects (events, entities and processes). The cyberspace-time reference frame primarily comprises of three spatial dimensions plus time. The coordinates are required to uniquely identify cyberspatial object. Subsequently, a cyberfield may be established as a byproduct of the cyberspatial entity network. The formation and evolution of the cluster of the entities is govern by the rules/protocols.

- Cyberspatial object: Three types of cyberspatial entities are considered. Logical entities, existing only in virtue of demarcations induced by human cognition and actions, such as applications, virtual entities, or simulated objects. Physical entities are tangible entities determining a possible pattern of information flow and the typical operations that can be made. Lastly, well-defined data as an entity, which can be in form of event or processes.

- Existence: Existence of spaces (physical space, logical space, information space, and cyberspace) 
and the cyberspatial objects is defined. As existence deprived of mathematical rigor is just meaningless, existence of entity is partly justified, for instance, by the spatial coordinate.

The general relation between the discussed ontological concept of cyber philosophy is given in Figure 1.

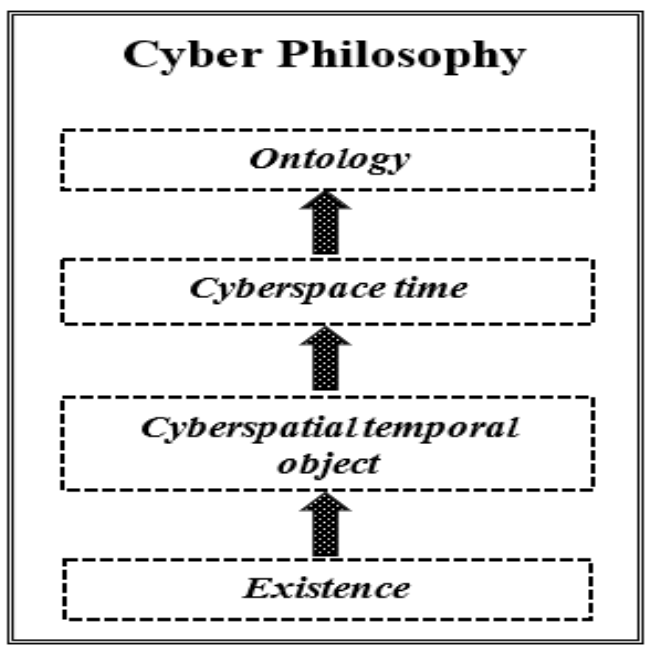

Figure 1: Ontological concept of Cyber Philosophy

\subsubsection{Cyber Science}

Cyber science studies cyberspatial entities forming systematic information manifold.

- Network science: Communication networks which enable entities interaction with each other are the core of the cyberspace fabric. This fabric is better understood using theories and methods including graph theory, statistical mechanics, data mining and information visualization, and social structure from sociology field. A complex problem is defined in graph theory and network science techniques to get a feasible solution or improve performance such as the work in [41]

- Topological dynamics: The ultimate focus of network modeling is designing an efficient protocols/rules, incorporating many dynamical characteristics observed in the real networks. Cyberspace is network of networks [17] and the topological structure and dynamic behavior of its entities are appropriately modeled, and many useful analyses can be made. For instance, the router and/or autonomous system topology haven been found to have some defined properties such as hierarchical structure, high clustering, presence of hub, average shortest path etc. [18], [19].

- Rules/principles: Network is driven by the rules and principles, the search of which has led to series of quest from random network [20] to heuristically optimized trade-off [21], [22] models of technological network. An optimization as in [40].

- Interaction: The mentioned topological structure and dynamics are formed conceptually from individual entities, to pairwise interactions, to local structures, and eventually to the whole network system. The interactions among entities and between different levels generate many unexpected or unpredictable behaviors, such as power law degree distribution emergence and chaos [20][22].

The Figure 2 summarizes the relations of what constitutes cyber science of entities formation and interaction.

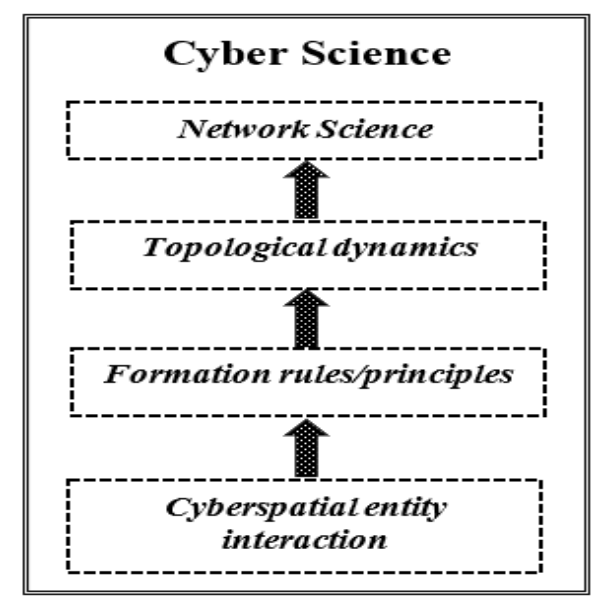

Figure 2: Topological formation and interaction of entities

\subsubsection{Cyber Information Technology}

To make sense of information (another cyber entity), information theory concepts could come handy. Shannon's information theory describes measures for conditional events, streams of messages, and situations. This work is the basis for contemporary information and telecommunications systems, including, telephones, world wide web (www) and the Internet. Computable predictions for cyberspace dynamics could be better explained when related to field of information:

- Information theory: Theory of information suggest that information can be as simple as a change in state. The key elements in communication system of cyberspace (the source, channel, and receiver) are nothing more than the cyberspatial entities-a finite set of information units.

- Information syntax and semantics: An instance of information as semantic must consist of meaningfully well-formed data. The mathematical theories of communication are applied to the data, and the philosophical theories are applied to the semantics, also known as the content. Information as being encoded, transmitted, and stored is non-negative and additive. The key is using mathematical theory of communication to give a precise meaning to information instead of using information as an ordinary sense as thoughts process-used in paraspace notion of cyberspace.

- Information exertion like an energy: Energy is considered from an angle of physical, conceptual 
and social state change is the most fundamental sense of information. The energy is what influences changes in systems at all levels. Information in this context is considered as a kind of an energy that governs the changes in physical or conceptual states. Energy has basic properties and measures and is well understood through the quality and quantity of changes it induces.

Cyber Information Technology, as sum up in Figure 3, perceive information as an entity exerted like an energy among cyberspatial objects.

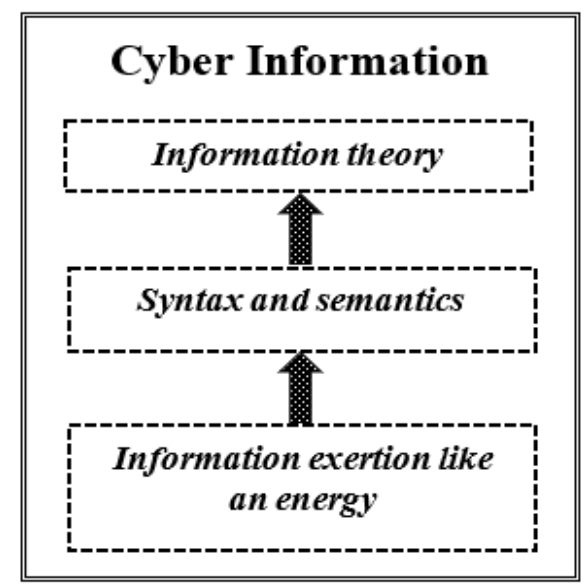

Figure 3: Information like a force exerted among cyberspatial entities

The formal conception of cyberspace is at the intersection of cyber science, cyber information technology science, and cyber philosophy which bridged by Cyber logic [11]. More specifically, the existence of space and spatial entities governed by a topological rule and instructional information that the entities used to changes theirs states and the state of other connected entities. This is summarized in Figure 4.

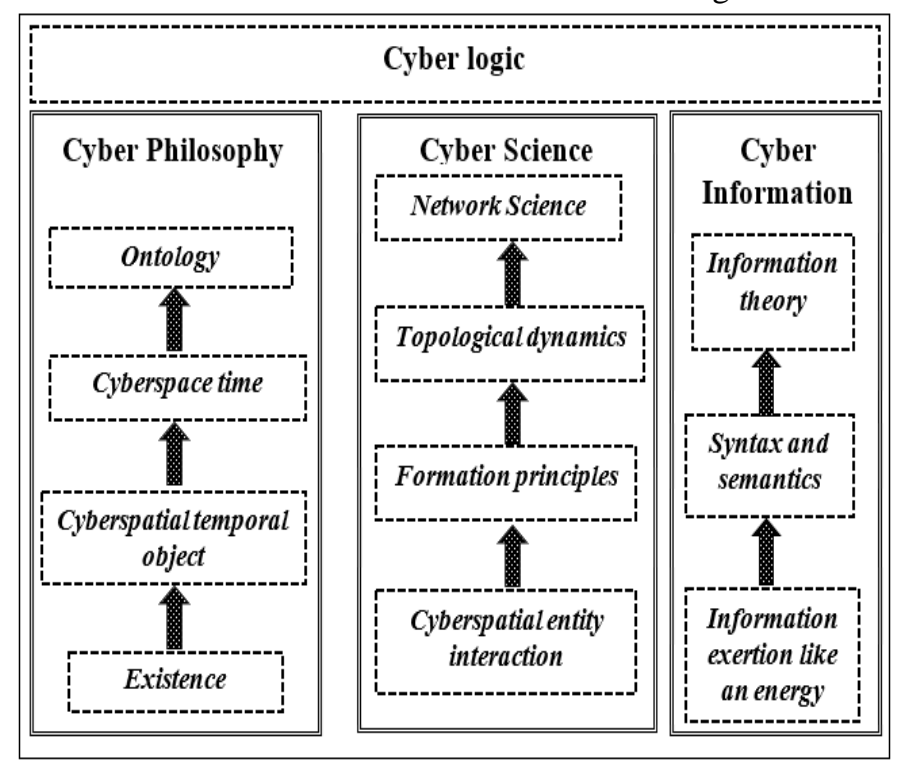

Figure 4: Cyberspace disciplines

\subsection{Definition}

Variety of cyberspace definitions are given in literature and mostly posit that the core of cyberspace consists of connected networks of hardware, software and data. It is also known that the relationship between these entities is complex and heterogeneous. This highlight the fabric, referring to the cyber, part of cyberspace.

However, "space" concept is paramount as well. To define something as a space, a corresponding topology or metric needs to be formulated [23]. By metric, it implies that the distance measure between entities is explicitly considered so that the corresponding axioms are met [24]. Depending on the underlined spatial theory, different calculations of a distance in cyberspace is possible. For instance, shortest path between two nodes on a graph from graph theory.

Convergence of many other spatial concepts are being coined. For instance, mobile cyber-physical system (MCPS), cyber-physical-social system (CPSS), and cyber physical-social-thinking hyperspace. Physical space is the real-world inhabited by real object, the social space is of social relation of human activities and the thinking space is a space of thoughts and intellectual activities. For a meaningful formal description, we replace social space with logical space, and thinking space with information space. Physical space is the place where physical infrastructure and devices for cyberspace exist; the logical space is the reference of various rules governing the interaction of the networked entities of cyberspace; and respectively, the information space is a representation of knowledge in a conceptual space. These cyber-enabled spaces are formalized as a unified cyberspace and spatial temporal dimensions. Cyberspace occupied by cyberspatial entities which are distinguished by their spatial coordinates and dynamics in time.

A typical example of cyberspatial entity is the cyber physical system, integrating cyberspace with the physical world and responsible for governing physical systems whose effects unfold in cyberspace. There may also be functionalities and events involving relationship between cyber entities and their surroundings. Figure 5 shows the paradigm shift to formal cyberspace concept from the generalized cyberspace. 


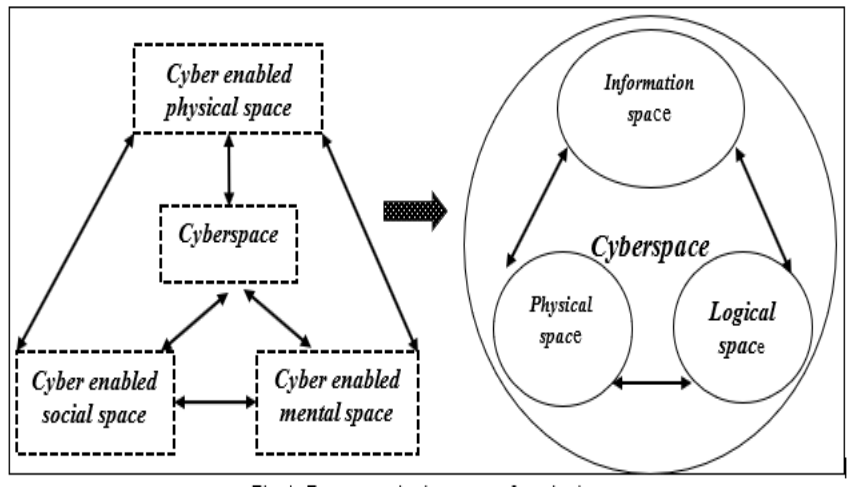

Figure 5: From general cyberspace to formal cyberspace.

The spaces are integrated into cyberspace by coupling data/information, logical cyber interactions, space-time or geospatial manifold. Therefore, the Cyberspace has the following characteristics:

(i) Integration: Cyberspace as a combination of variety of spaces.

(ii) Interconnection: Cyberspace related with other spaces.

(iii) Interaction: Cyberspace can influence other spaces.

(iv) Interpretation: Cyberspace may be considered in a meaningfully rigorous perspective.

Hence, we propose a formal cyberspace as:

A manifold of cyberspatial entities, whose behavior unfold in cyberspace time with the topological interactions that are in turn governed by network principles such as optimization driven by an information exerted and transmitted among the entities.

\section{CYBER PHILOSOPHY: EXISTENCE IN CYBERSPACE-TIME}

\subsection{Existence:}

One of the defining characteristics of matter is to occupy a space and a physical existence points to a substance made up of matter and/or energy. Varieties of cyber entities exist, many of which directly correlate to entities in the physical worlds. As result, the real entities have a cyber-existence and are interconnected.

\subsubsection{Space}

Space is a collection of infinitesimally small places/points/locations where entities may be found. Similarly, Cyberspace is taken as having objects coordinates just as it has been considered as 'parallel' universe [25], and that "physical space and cyberspace interpenetrate" [26]. "Space, is a boundless, three-dimensional extent in which objects and events occur and have relative position and direction." [27]. It consists of some objects that are treated as points, and some relationships between these points. Therefore, we need to specify relevant coordinates, dimensions, objects and their relationships to give the spatial meaning. Volume, locus, or destination can be implied in cyberspace as it is simultaneously physical; tangible and real, and present in geospace (G), informational; both logical and virtual, and present in info-space (I), and social; organizational and political, and present in socio-space (S) [16].

- Physical space: Cyberspace viewed as an entity embedded in physical space and the very existence of cyberspace in physical space is thus investigated. Cyberspace is physical, present in geospace and shares characteristic of physics. The classical three-dimensional Newtonian space-time framework embodied physical cyberspatial objects operating in geospace. The geocentric coordinates, which are system of locating object in three-dimensions such as latitude, longitude and altitude, could also specify the physical location of cyber object at a particular time.

Consider a 3-D manifold, $\mathbb{R}^{3}$, and let $\epsilon_{i}$ and $P_{i}^{P}\left(t_{k}\right)$ be cyber object; such as cyberphysical system, and its physical (geospatial) location at time $t$ respectively. The two indexes required are (i) the geocentric coordinates, a system of locating an object in three-dimensions along latitude, longitude and altitude $(\mathrm{x}, \mathrm{y}, \mathrm{z})$; (ii) the spherical coordinates defined by using radial, azimuth and zenith angles, $r, \theta$ and $\varnothing$ respectively. Figure 6 shows this geocentric coordinate.

$$
P_{i}^{G}\left(t_{k_{k}}\right)=\left\{x_{i}, y_{i}, z_{i}\right\}\left(t_{k_{k}}\right)[16]
$$

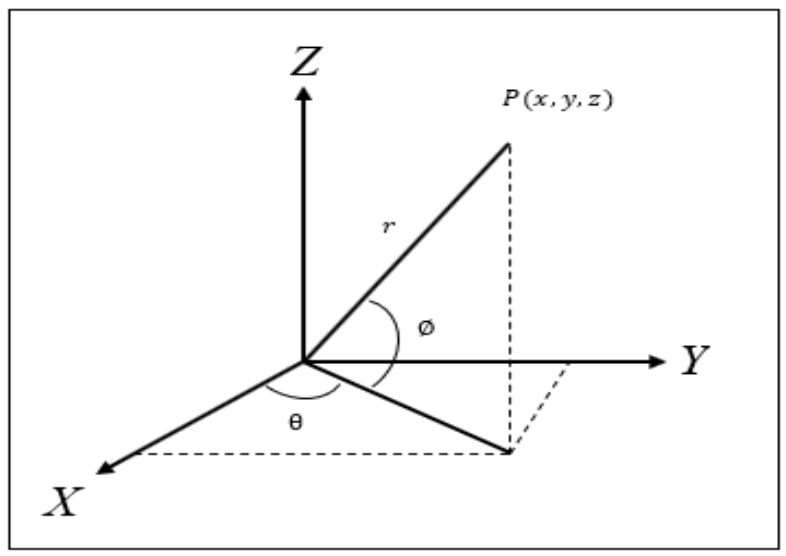

Figure 6: Cyberspace disciplines

Alternatively, the discrete partition model of physical space, for instance a digital earth, could be used to locate cyber object at multiple resolution. For example, Geodetic Discrete Global Grid Systems (GDGGS) [28] or Digital Earth Reference Model (DERM) [29] allow mathematical operations to be defined on the index, where $\{x, y, z\}$ refers to the location of a hexagonal region defined by a tessellation on the Earth's surface. 
The addressing of an object, using geospatial address ( $g s a$ ), the Pyxis digital earth index, is given in [30] as:

$$
g s a=<\text { dga: dra: dea }>
$$

Where: dga: is global address specifying resolution 1 index "AN", dra: is the Pyxis DERM resolution address which specifies the higher resolution (>1) indices "N...N.". and dea: is the Pyxis DERM elevation address representing the thickness (volume) of the cell identified by " $<d g a \equiv d r a>$ " [30].

- Logical space: A network (formally as graph) is an abstraction of spatial relationships, represented by connectivity between spatial entities. It is an ideal method that capture the structure of the internet. Nodes represent an object such as computer, router, Local Area Network (LAN), an autonomous system, web documents etc. Connectivity among nodes is depicted accordingly by an edge. In this way it is possible to identify and describe some part or an entire underlined topology of cyberspace in an appropriate way. For example, let $\mathrm{V}(\mathrm{G})$ be a given set of points/nodes/entities. Then a topological space is a collection of subsets of $V(G)$, such that every point in $\mathrm{V}(\mathrm{G})$ has some neighbors and the states are influenced by their interaction.

Mapping of entities can be done at different levels of topology. It is possible to describe the network by using router adjacencies. At much higher level, the Internet is mapped in a graphical space from autonomous system routing path information. However, many factors may influence the topology. For instance, where new connections could emerge with higher probability. Empirical analysis shows that location of routers and autonomous system are related with population density [30].

The basic unit of logical space is a pair of points or logical location of entities and associated with states or values. Formally, the topology space is a function from the set of nodes, determined by its location, to the set of states defined by:

$$
\begin{gathered}
f: E \rightarrow S \\
X=\left\{\left(\theta_{\mathrm{i}}, x\left(\theta_{\mathrm{i}}\right): \theta_{\mathrm{i}} \in E\right\}\right.
\end{gathered}
$$

Where $\epsilon_{i} \in \mathrm{E}$ is an entity (cyberspatial object at position) and $x\left(\theta_{i}\right) \in \mathrm{S}$ is a state/value. A generalized case is where a node takes more than one attribute/state simultaneously in a multi-variate situation. In this case the set of states is the set product of these values, and therefore:

$$
S=\prod_{i=1} S i
$$

Vertically an underlying hierarchy is identified in a logical structure of cyberspace. The network partitioned into autonomous systems that varies in size and functions and correspond to different backbones and Internet Service Providers (ISP) - providing connectivity at national, inter-continental, regional and local level. A local autonomous system, L, is a subordinate to, and therefore dependent on national autonomous system, $\mathrm{N}$, which is superior to, and therefore responsible for its subordinates including regional autonomous system, R. We can identify a logical space, as a framework to specify the location of logical object with respect to its administrative or connectivity role within one or more autonomous systems. Let $P_{i}^{L}=\left\{n_{i}, r_{i}, l_{i}\right\}$ be the spatial position of logical entity $P_{1}^{L}$. Figure 7 shows the logical coordinate of an entity in 3-dimensions.

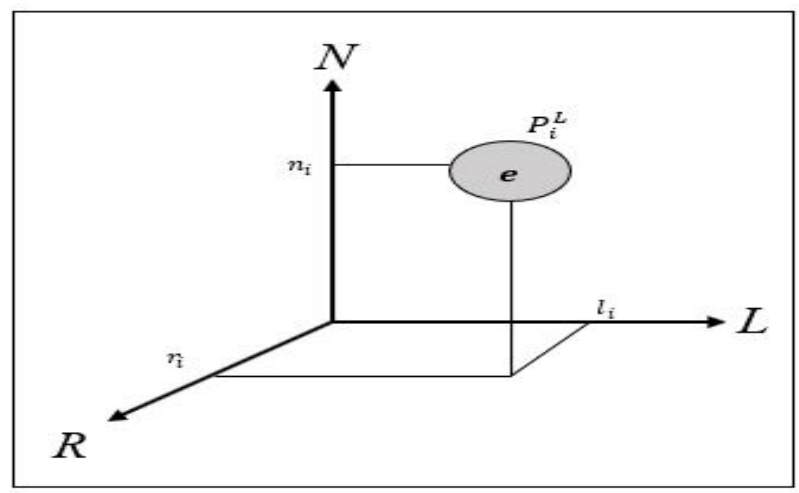

Figure 7: Logical coordinate of an entity.

- Informational space: A conceptual space can be constructed by processing the data matrix of the observations based on the variable values and class labels [31] - A conceptual space is a geo-metrical structure which is defined by a set of quality dimensions. The quality dimensions represent the features of objects in the space based on their measured quality values. One conceptual space can consist of multiple domains. A domain in the conceptual space is represented as a set of interdependent quality dimensions which are logically integrated." [31]. The conceptual space models the attributes of concepts for a comprehensive reasoning, and useful as a framework for content determination using semantic inferences. Fuzzy representation of conceptual spaces' elements by integrating conceptual spaces theory with the topic of computing with words is possible [32].

Conceptual spaces theory presents a framework for cognitively meaningful attributes in various domains within a geometrical structure in order to model, categorize, and represent the concepts in a multi-dimensional space [34]. The theory of conceptual spaces is a knowledge representation framework exploring how different information can be formalized, both from a psychological point of view and for developing an artificial system [35].

From [31], [32], [33],[34] conceptual information space I is considered as a 4-tuple $\langle\mathrm{Q}, \Delta, \mathrm{C}, \Gamma\rangle$, where $\mathrm{Q}$ is a set of quality dimensions which is the framework used to assign properties to objects and to specify relations among them, $\Delta$ is a set of domains, $C$ is a set of concepts in the space $I$, and $\Gamma$ is a set of instances representing the concepts. 
Similarly, we have a framework in which to specify the locations of informational object's location, specifically service access points (SAPs) or the communications ports. Let $P_{i}\left(t_{k i}\right)=\left\{g_{i}, s_{i}, G_{i}\right\}\left(t_{k i}\right)$ be the info spatial location of $\varepsilon_{i}$ nth service access point (SAP) at time $\left(t_{h}\right)$; where $g$ is a global network address, $s$ is the subnetwork address and $a$ is the subnetwork's SAP [7]. The Communication system interact through messages as a function of time and other variables defined in a dimensional continuum.

\subsubsection{Cyber spatial entity}

- Cyberspatial entities (events, agents or processes) are objects whose behaviors unfold in cyberspace. These objects in physical space, logical space or info space require corresponding indices to be distinguished from one another and for a given object, one state from another [29]. Therefore, cyberspace is occupied by discrete identifiable entities, each within a spatial reference frame.

- Cyber physical object existence: In physical space there exist many entities that can be identified and formalized. For instance, cyber physical system is "internetworked information systems responsible for governance of physical processes" [7]. These entities can be identified by their coordinates, geospatial dimension, and are here formalized in such a way that each object has unique identity (I), spatial embedment (S), and attributes $\left(A_{n}\right)$. Therefore, a cyberspatial physical object or entity $P_{i}^{G}$ has at least 3-tuples $\left\{i_{i}, s_{i}, a_{i}\right\}$.

The spatial embodiment $S \in \mathbb{R}^{2}$ is the geospatial indices and serve as the object's identity required to distinguish objects. The state of an object is a collection of attributes describing object's static properties.

$$
\sum_{n=1}^{N} A_{n} \leq 1
$$

Equation 6 states that at most one of the properties of $A_{n} \in\left\{A_{1}, A_{2}, A_{3}, \ldots A_{n}\right\}$ should hold at any a time.

- Cyber logical object existence: There are many logical entities which are usually represented as a node in the network, in the cyberspace. Consider $e_{i} \in \mathrm{E}$ as an entity such as services, application or web documents. In the topology of logical space, we have $e_{i} \in \mathrm{E}$ and $\mathrm{E} \neq \emptyset$.

- Cyber informational object existence: Data is an example of information entity. Information can be quantified using a fundamental unit, Shannon entropy which is a measure of the information in a message. The Shannon entropy of a variable $\mathrm{X}$ is defined as:

$$
H(x)=-\sum_{x=1}^{N} P(x) \log _{2} p(x)
$$

Where $p(x)$ is the probability that $\mathrm{X}$ is in the state $\mathrm{x}$, and $p \log _{2} p$ is considered 0 if $p=0$. [36]

Information in form of a message is defined as $m_{i, j}=\left\{\theta_{i}, \theta_{j}, n, l, t_{h}\right\}$. Sent from entity $\theta_{i}$ to $\theta_{j}$; for a payload I with a particular action/service selector, $n \geq \mathbf{1}$ and "message sent" time $t_{k}$ [7].

Consider $\mathrm{M}$ as the set of all messages $\left[\mathrm{m}^{1}, \ldots, m^{w}\right]$ possible for $\mathrm{X}$, and $p(x)$ as the probability of some $x \in M$, then the entropy of $\mathrm{X}$ would be defined as $\boldsymbol{H}(\boldsymbol{X})=\boldsymbol{E}_{x}\left(\boldsymbol{Z}\left(x^{\prime}\right)\right)$ where $I(x)$ is the entropy contribution of an individual message. [36].

\subsection{Cyberspace time}

Because the traditional perception of space attributed to cyberspace does not amount to meaningful concept, the basis of cyberspace in contemporary physics is examined. As a parallel universe of information and telecommunication devices, cyberspace as an abstract, geometrical, and mathematical field in which data structures are built. This is a proposition that cyberspace may be more accurately described as a part of the physical universe. Considering some similar physics concepts to describe cyberspace mechanics and cyberspacetime [7]. Although this leads to many questions than answers, the existing theories could be utilized.

A cyberspatial objects' state requires description in either or both of geospatial, logical space or and info spatial terms. For dynamic cyberspatial object its behaviors unfold in both cyberspace and time. Like the structure of space time, cyberspace time is thus characterized geometrically by a tuple C:

$$
\mathbb{C}=(\mathrm{C}, \mathrm{L}, \Omega, \mathrm{V})
$$

Whereas $\mathrm{C}$ is assumed Euclidian and compact of primarily 3 dimensions (Physical $(P F)$, logical $\left(P_{t}\right)$, and information $(P /))$ plus time. $\mathrm{L}$ is a connection on $\mathrm{C}, \Omega$, as a differentiable 1 -form field on $\mathrm{C}, \mathrm{V}$ is as vector field on $\mathrm{C}$. Such that each point of the manifold $\square$ is an entity or an "event" which is characterized by their instant and point in time and place of occurrence.

\section{CYBER SCIENCE: TOPOLOGY AND DYNAMICS}

Spatial interaction is explained by the influence/connection and information flow among entities, nodes in graph. This is locally represented using relational topology and globally using meta-relational topology. The information with regards to the local topology at a given time $t$ is then referenced in a matrix $\dot{M}(\mathrm{t})$ of ExE. For example, a function from the location set $\mathrm{E}=\left\{e_{1}, e_{2}, e_{3}, e_{4},\right\}$ to the value set $\mathrm{V}=\{1, \mathrm{O}\}$. To represent a given spatial relations, including an arbitrary set of neighborhoods, we have meta-relational topology $\mathrm{T}$ as a topology where relational topology is associated with each $e_{i} \in \mathrm{E}$. A relational topology, $T_{n}$, is the set of all those entities in 
E which influence node $e_{i}$. Then $\mathrm{T}$ can be represented by a binary matrix on $\mathrm{E}$, in which all influencing nodes have value 1,0 .

Similar to [37] we defined the meta-relational topology as:

$$
\begin{gathered}
T=\left\{\left(e_{i}, T_{i}\right): \epsilon_{i} \in E\right\} \\
T_{i}=\left\{\left(e_{i}, t_{i}\right) \mid t_{i}\left(e_{i}\right) \in\{0,1\} \forall \epsilon_{i} \in E\right\}
\end{gathered}
$$

Table 1 summarizes the relation between the graph concepts and topological dynamics in the logical space of the cyberspace manifold.

Table 1: Graph space and logical space

\begin{tabular}{|cc|cc|}
\hline \hline Graph & & Logical space & \\
\hline \hline \multirow{2}{*}{ Elements } & & Elements & \\
& & Cyber entities & $\mathrm{E}$ \\
\hline Vertex set & $\mathrm{V}(\mathrm{G})$ & Meta relational topology & $T$ \\
Edge set & $L(G)$ & Entities state & $S$ \\
States & $S$ & Relational topology & $T_{n}$ \\
Neighborhoods & $N$ & & \\
& & & \\
\hline \hline
\end{tabular}

\section{- Entity interaction:}

Dynamic graphs representing the physical connectivity as physical location may provide a vital information in research that correlate the connectivity and performance with the real physical distance among routers [30].

For logical interaction, the entities defined as a node, for example a service or a software application running in an enterprise system with link representing pair-wise relationships between them. The relationship between physical entity and logical entity is clear in this regardServices that run on a given cyber physical system. From a network perspective, the topological connectivity of entity in logical space is different from those at the physical.

With regards to information entity communication, we also have information in form of a network. For instance, the WWW is a universe of information formed by linking resources for easier accessibility [38].

The interactions among different entities is either homogeneous (entities considered with respect to specific spatial domain), or heterogeneous (entities from different spatial domain). In essence, the model can uniformly describe homogeneous and heterogeneous entities interacting with their complex relationships in multi-dimensional transdisciplinary cyberspace. This complex interaction could be represented, from a graph theory, for instance as hyper graph $\mathbf{H}(\mathbf{V}, \mathbf{E})$ [39], in which further research needed to be done.

\section{CONCLUSION}

Cyberspace definition and delimitation problems in theory are better addressed through formalized cyberspace. We examined the empirical and boundless perceptions of cyberspace shaped by its ubiquitous evolution. A transdisciplinary integration of concepts of formal cyberspace base on three fundamental aspects in three disciplines (philosophy, Science and information theory) has been proposed- The existence of spaces and entities, dynamic interaction and topology, and information as an energy. It was suggested that that cyberspace is a manifold of cyberspatial entities, whose behavior unfold in cyberspace time with the topological interactions that are in turn governed by network principles such as optimization driven by an information exerted and communicated among the entities.

Considering the evolving concepts of cyberspace and multidisciplinary knowledge spectrum, three key issues towards theoretic view of cyberspace are vital:

- Cyberspace time: An existence of spatial framework to identify, represent, model, and characterize various types of cyberspatial entities and cyberspace manifold.

- Interaction: Homogenous and heterogeneous connections between the entities, giving the topological dynamics of the manifold.

- Information theory as a meaningful basis to replace mental space conception of cyberspace.

We, therefore, suggest a paradigm shift to advance the theory development process by identifying aspects of existing domain theory that inform cyber theory development.

\section{REFERENCES}

1. Madnick, Stuart; Choucri, Nazli; Camiña, Steven; and Woon, Wei Lee, "Towards Better Understanding Cybersecurity: or are "Cyberspace" and "Cyber Space" the Same?" WISP 2012 Proceedings. 27, 2012 http://aisel.aisnet.org/wisp2012/27 https://doi.org/10.1080/10570319909374648

2. Strate $L$. The varieties of cyberspace: Problems in definition and delimitation. Western Journal of Communication (includes Communication Reports). 1;63(3):382-412, Sep 1999

3. Ning, Huansheng, Xiaozhen Ye, Mohammed Amine Bouras, Dawei Wei, and Mahmoud Daneshmand. "General cyberspace: Cyberspace and cyber-enabled spaces." In IEEE Internet of Things Journal 5, no. 3, 1843-1856, 2018

4. H. Ning, H. Liu, J. Ma, L. T. Yang, and R. Huang, "Cybermatics: Cyber-physical-social-thinking hyperspace based science and technology,"Future Generation Computer Systems, vol. 56, pp. 504-522, 2016. https://doi.org/10.1016/j.future.2015.07.012

5. J. Ma, "Cybermatics for cyberization towards cyber-enabled hyperworlds," in Mobile Cloud 
Computing, Services, and Engineering (MobileCloud), 2016 4th IEEE International Conference on. IEEE, 2016 pp. 85-86.

6. J. Ma et al., "Perspectives on Cyber Science and Technology for Cyberization and Cyber-Enabled Worlds," In IEEE 14th Intl Conf on Dependable, Autonomic and Secure Computing, 14th Intl Conf on Pervasive Intelligence and Computing, 2nd Intl Conf on Big Data Intelligence and Computing and Cyber Science and Technology Congress Auckland, pp. 1-9, 2016, https://doi:10.1109/DASC-PICom-DataCom-CyberSciT ec.2016.17.

7. Bayne, Jay S. "Cyberspatial mechanics." IEEE Transactions on Systems, Man, and Cybernetics, Part B (Cybernetics) 38, no. 3 (2008): 629-644.

8. Sun YC, Song HB, Jara AJ, et al. Internet of things and big data analytics for smart and connected communities. IEEE Access 2016; 4: 766-773.

9. Wang, Jinfa, Hai Zhao, and Xiao Liu. "Research on life characteristics of Internet based on network motifs." arXiv preprint arXiv:1611.01361 (2016).

10. Gozzi, Raymond. "The cyberspace metaphor." ETC: A Review of General Semantics 51, no. 2 (1994): 218-223.

11. H. Ning, Q. Li, D. Wei, H. Liu, and T. Zhu, "Cyberlogic paves the way from cyber philosophy to cyber science," IEEE Internet of Things Journal, 2017 https://doi.org/10.1109/JIOT.2017.2666798

12. Bryant, R., (2001). "What kind of space is cyberspace" Minerva-An Internet journal of philosophy, 5, pp.138-155.

13. McCarthy, T.D., (2012) Traveling Domain Theory: A Comparative Approach for Cyberspace Theory Development (Doctoral dissertation, FLETCHER SCHOOL OF LAW AND DIPLOMACY (TUFTS UNIVERSITY)).

14. Delany, S.R., Is cyberpunk a good thing or a bad thing? Mississippi Review, 47(48), pp.28-35, 1988

15. Moor, J.H. and Bynum, T.W., 2002. Introduction to cyberphilosophy. Metaphilosophy, 33(1/2), pp.4-10.

16. Koepsell, David R. "The Ontology of Cyberspace: Preliminary Questions," paper presented at the Tri-State Philosophical Association Meeting, St." Bonaventure University 22 (1995).

17. Chen, Guanrong, Xiaofan Wang, and Xiang Li. Fundamentals of complex networks: models, structures and dynamics. John Wiley \& Sons, 2014.

18. Fan, Z.P. (2006) Complex Networks: From Topology to Dynamics. PhD Thesis, City University of Hong Kong, May.

19. Waxman, B-M. Routing of multipoint connections. IEEE Journal of Selected Areas in Communication, 6(9): 1617-22, 1988

20. A. Fabrikant, E. Koutsoupias, and C. H. Papadimitriou. "Heuristically Optimized Tradeoffs: A New Paradigm for Power Laws in the Internet." In Proceedings of the 29th International Colloquium on
Automata, Languages, and Programming, Lecture Notes in Computer Science 2380, pp. 110-122. BerlinHeidelberg: Springer-Verlag, 2002

21. Alderson, David, Walter Willinger, Lun Li, and John Doyle. "An optimization-based approach to modeling internet topology." In Telecommunications Planning: Innovations in Pricing, Network Design and Management, pp. 101-136. Springer, Boston, MA, 2006.

22. Siganos, G., Faloutsos, M., Faloutsos, P. and Faloutsos, C. (2003) Power laws and the AS-level Internet topology. IEEE/ ACM Transactions on Networking, 11(4): 514-24 https://doi.org/10.1109/TNET.2003.815300

23. Deza E. and Deza M.M. (2006) Dictionary of Distances. Amsterdam, Elsevier.

24. Zhu, Yueqin, Yongjie Tan, Ruixin Li, and Xiong Luo. "Cyber-physical-social-thinking modeling and computing for geological information service system." International Journal of Distributed Sensor Networks 12, no. $11,2016$.

25. Wellman B. (2001) Physical place and cyberspace: the rise of personalized networking. International Journal of Urban and Regional Research, 25(2): 227-252.

26. Space, (1998)."Space - Physics and Metaphysics". Encyclopædia Britannica.

https://www.britannica.com/science/space-physics-andmetaphysics Archived from the original on 6 May 2008. Retrieved 17 june 2019

27. Sahr, K., White, D., and Kimerling, A.J., Geodesic Discrete Global Grid Systems. Cartography and Geographic Information Science, 30(2), pp 121-134, 2003.

28. Vince, Andrew, and Xiqiang Zheng. "Arithmetic and Fourier transform for the PYXIS multi-resolution digital Earth model." International Journal of Digital Earth 2, no. 1 pp 59-79, 2009.

29. Bayne, Jay S. " Agent-Based Modeling of Complex Spatial Systems." Workshop on Agent-Based Modeling of Complex Spatial Systems, April 14-16, 2007

30. Yook, S., Jeong, H. and Barabasi, A-L. (2002) Modeling the Internet's large-scale topology. Proceedings of the National Academy of Sciences, 99(21): 13382-13386 https://doi.org/10.1073/pnas.172501399

31. Aisbett, Janet, John T. Rickard, and Greg Gibbon. "Conceptual spaces and computing with words." In Applications of Conceptual Spaces, pp. 123-139. Springer, Cham, 2015.

32. Bechberger, Lucas, and Kai-Uwe Kühnberger. "A thorough formalization of conceptual spaces." In Joint German/Austrian Conference on Artificial Intelligence (Künstliche Intelligenz), pp. 58-71. Springer, Cham, 2017

33. Gärdenfors, P. (2000). Conceptual Spaces: The Geometry of Thought. MIT press, 2000.

34. Gärdenfors, P. (2004). Conceptual spaces as a framework for knowledge representation. Mind and Matter, 2 (2), 9-27. 
35. Floridi, L., 2005. Semantic Conceptions of Information. First published Wed Oct 5, 2005; substantive revision Fri Jan 28, 2015. Date accessed 15.05.2019

https://plato.stanford.edu/entries/information-semantic/

36. Shannon, C. (1948). A Mathematical theory of communication, Bell SystemTechnical Journal,Vol. 27, p. 379-423, 623-656. DOI: 10.1145/584091.584093 45, 47

37. Takeyama, M. (1997). Building spatial models within GIS through Geo Algebra. Transactions in GIS, 2(3), 245-256

38. Albert, R., Jeong, H. and Barabási, A-L. (1999) Diameter of the World-Wide Web. Nature, 401: 130-1

39. Peng, C. (2016). Research and Construction Model of Cyberspace Based on Hypergraph. In 5th International Conference on Advanced Materials and Computer Science (ICAMCS 2016). Atlantis Press.

40. Melnyk Yurii et al., (2019). The Process of Network Flows Distribution based on Traffic Engineering Method. International Journal of Advanced Trends in Computer Science and Engineering. 8(6): 3036-3042. https://doi.org/10.30534/ijatcse/2019/60862019

41. Shanmuk Srinivas Amiripalli et al., Performance improvement model for Airlines connectivity system using Network Science. International Journal of Advanced Trends in Computer Science and Engineering, 9(1), January - February 2020, 789 - 792 https://doi.org/10.30534/ijatcse/2020/113912020 